\title{
Capture versus suppression of attention by salient singletons: Electrophysiological evidence for an automatic attend-to-me signal
}

\author{
Risa Sawaki AND STeven J. Luck \\ University of California, Davis, California
}

\begin{abstract}
There is considerable controversy about whether salient singletons capture attention in a bottom-up fashion, irrespective of top-down control settings. One possibility is that salient singletons always generate an attention capture signal, but this signal can be actively suppressed to avoid capture. In the present study, we investigated this issue by using event-related potential recordings, focusing on N2pc (N2-posterior-contralateral; a measure of attentional deployment) and Pd (distractor positivity; a measure of attentional suppression). Participants searched for a specific letter within one of two regions, and irrelevant color singletons were sometimes present. We found that the irrelevant singletons did not elicit N2pc but instead elicited Pd; this occurred equally within the attended and unattended regions. These findings suggest that salient singletons may automatically produce an attend-to-me signal, irrespective of top-down control settings, but this signal can be overridden by an active suppression process to prevent the actual capture of attention.
\end{abstract}

The world presents too much information to be perceived, remembered, and acted upon, and we rely on two classes of attentional control mechanisms to bias processing toward the most important information. One is the set of top-down attentional mechanisms that control attention in favor of current goals. The other is the set of bottomup attentional mechanisms that control attention on the basis of the properties of the stimuli themselves (Corbetta \& Shulman, 2002; Desimone \& Duncan, 1995; Egeth \& Yantis, 1997; Kastner \& Ungerleider, 2000; Theeuwes, 1991a; Yantis \& Egeth, 1999).

A debate has arisen concerning to what degree top-down mechanisms can attenuate capture of attention by salient singletons (i.e., stimuli that contain a unique feature value in an otherwise homogeneous scene; see Bacon \& Egeth, 1994; Folk \& Remington, 1998, 1999; Folk, Remington, \& Johnston, 1992; Folk, Remington, \& Wright, 1994; Hickey, McDonald, \& Theeuwes, 2006; Lamy \& Egeth, 2003; Sawaki \& Katayama, 2008, 2009; Theeuwes, 1991b, $1994,2004)$. The findings of previous studies have led to differing hypotheses of whether attentional capture by salient singletons can be purely stimulus-driven or whether it is always modulated by top-down attentional settings induced by task demands. ${ }^{1}$

The bottom-up saliency hypothesis proposes that attention initially shifts to the most salient item in the visual field. By this account, attentional capture is purely stimulus driven, with no influence of task goals (Hickey et al., 2006; Theeuwes, 1991a, 1992, 2004). Evidence for stimulus-driven capture has been found in many studies in which a color singleton is presented together with a less salient target defined by shape. Even though color is not task relevant, the presence of a color singleton delays target detection, suggesting that color singletons capture attention in a bottom-up fashion (e.g., Theeuwes, 1991a).

The bottom-up saliency hypothesis further proposes that top-down goals can influence capture by modulating the allocation of spatial attention (Theeuwes, 1991b; Theeuwes \& Burger, 1998; Yantis \& Jonides, 1990). When spatial attention is highly focused on the location where a target is expected, irrelevant salient singletons can be ignored if they are presented outside the focus of attention (Theeuwes, 1991b), presumably because the suppression of perceptual processing outside the focus of spatial attention attenuates the salience of the singleton. This modified version of the bottom-up saliency hypothesis still maintains that salient singletons capture attention in a bottom-up fashion within the attended area: Spatial attention can prevent stimulus-driven capture within the unattended area, but salient singletons automatically capture attention irrespective of the participant's goal within the attended area (e.g., Belopolsky, Zwaan, Theeuwes, \& Kramer, 2007; Theeuwes, 2004).

A well-known alternative to the bottom-up saliency hypothesis is the contingent involuntary orienting hypothesis, which proposes that the capture of attention by salient

R. Sawaki, rsawaki@ucdavis.edu 
singletons depends on task-dependent attentional control settings that determine what sorts of stimulus properties will attract attention (Bacon \& Egeth, 1994; Folk \& Remington, 1998; Folk et al., 1992; Folk et al., 1994; Leber \& Egeth, 2006). For example, Folk et al. (1992) found that color singletons but not onsets attracted attention when the target was defined by color, whereas onsets but not color singletons attracted attention when the target was defined by onsets. In addition, Bacon and Egeth showed that when the target is defined by being a singleton in one dimension (i.e., a circle target among diamond nontargets), participants may adopt a strategy called singletondetection mode, in which they become sensitive to singletons in all dimensions. However, if the display contains multiple values along each dimension so that the target is not a singleton, the participants may adopt feature-search mode, in which they search for a specific feature. Bacon and Egeth found that singletons in an irrelevant dimension captured attention in the former case but not in the latter.

In these studies, slowing of target detection has typically been used as a measure of capture of attention by the irrelevant singleton, but this is an indirect means of assessing attention capture. Moreover, almost all researchers on attention capture have implicitly assumed that attention is a single process that is either captured or not captured by a given stimulus. However, abundant evidence indicates that multiple mechanisms of attention exist and that they operate at different processing stages under different conditions (for a review, see Vecera \& Luck, 2002). Both of these shortcomings of prior research have been addressed in recent studies of attention capture that included recordings of event-related potentials (ERPs) and, in particular, the N2pc component, which is a sensitive index of the covert deployment of visual attention (Eimer, 1996; Luck \& Hillyard, 1994a, 1994b; Woodman \& Luck, 1999, 2003). The N2pc component can be measured directly in response to the irrelevant singleton, avoiding the need to use changes in target processing to draw inferences about the capture of attention by the irrelevant singleton. Moreover, by using the N2pc component as a measure of attention, it is possible to ask whether the specific and well-characterized mechanism of attention reflected by this component is captured, rather than asking about some undifferentiated, generic mechanism of attention.

The N2pc component is observed as a more negative voltage at contralateral scalp sites relative to ipsilateral scalp sites relative to the position of an attended item in a visual search display, and it begins approximately $200 \mathrm{msec}$ after the onset of the stimulus presentation. The $\mathrm{N} 2 \mathrm{pc}$ component appears to be generated in intermediate and high levels of the ventral visual processing pathway, including area V4 and the lateral occipital complex (Hopf, Boelmans, Schoenfeld, Luck, \& Heinze, 2004; Hopf et al., 2000). It has been used by many researchers as an established electrophysiological marker of attentional allocation (Eimer \& Kiss, 2008; Hickey et al., 2006; Kiss, Jolicœur, Dell'Acqua, \& Eimer, 2008; Leblanc, Prime, \& Jolicœur, 2008; Lien, Ruthruff, Goodin, \& Remington, 2008; Rodríguez Holguín, Doallo, Vizoso, \& Cadaveira, 2009; Woodman \& Luck, 1999, 2003).
Hickey et al. (2006) found an N2pc to irrelevant color singleton stimuli in a task in which participants searched for a shape-defined singleton (a circle among squares or a square among circles, randomly intermixed). They concluded that attention was automatically captured by this color singleton, regardless of the top-down control settings. However, the participants were likely in singletondetection mode, making it impossible to assess whether the allocation of attention to the irrelevant color singleton solely reflected its bottom-up salience. In fact, subsequent $\mathrm{N} 2 \mathrm{pc}$ studies have supported the contingent involuntary orienting hypothesis by showing that attentional capture is dependent on current task settings and is mediated by whether salient distractors share the task-relevant feature (Eimer \& Kiss, 2008; Eimer, Kiss, Press, \& Sauter, 2009; Kiss et al., 2008; Leblanc et al., 2008; Lien et al., 2008). However, the same factors that have made it difficult to reach a resolution about the automaticity of singleton capture in behavioral studies also apply to the N2pc studies. The goal of the present study was to reexamine this issue in an experimental design that reflects the many lessons that have been learned from the behavioral studies of attentional capture. Specifically, to provide a maximally clear and unambiguous assessment of the automaticity of capture, it is helpful to follow the following principles.

First, to guarantee that participants are not using an attentional set that emphasizes singletons, the target should not be a singleton along any dimension. If the target is defined by being a singleton (e.g., a square among circles or a circle among squares, randomly intermixed), this inevitably biases the participants to search for feature discontinuities (i.e., to use singleton-detection mode). Even if the specific target feature is known in advance, the participants may still sometimes use singleton-detection mode to find it. If, for example, the target is always a circle among squares, the participants could in principle find the target via an attentional set that emphasizes curvature, but they might still use an attentional set that emphasizes singletons (especially if this is less effortful or more efficient than feature-based target detection). If, in contrast, the target was a circle but the shapes of the distractors were heterogeneous (e.g., a square, a triangle, an infinity symbol, etc.), task performance could not possibly be helped by an attentional set that emphasizes singletons. Thus, stronger conclusions can be drawn about automatic capture of attention by irrelevant singletons if the target itself is not a singleton.

However, it is also important to ensure that the targetdetection task is not overly difficult. If the task involves a serial search, attention might be narrowly focused, and the irrelevant singleton might fall outside of the focus of attention, in which case it may not capture attention (Theeuwes, 1991b, 2004). This leads to a second principle - namely, that the task should be designed in a manner that ensures that the irrelevant singleton is presented within an attended area of space.

A third principle comes from studies in which the irrelevant singleton occurs in a cue display that precedes the display containing the target (as in Folk et al., 1992). In these studies, it is possible that perceptual processing may 
be suppressed during the period of the cue display, leading to a lack of capture for the same reason that capture is absent when spatial attention is not focused on the spatial region occupied by the irrelevant singleton. Thus, to be able to interpret a lack of capture by an irrelevant singleton, it is advantageous to present the irrelevant singleton at a time when the participant expects the target to appear.

A fourth principle is relevant when ERPs are used to assess singleton capture: It is necessary to demonstrate that any ERP differences related to the singleton reflect attentional processes rather than a sensory imbalance between the singleton and nonsingleton items. Because lateral inhibition is often feature specific, neurons in areas V1 and V4 will produce a larger sensory response for singleton items than for nonsingleton items (Desimone \& Schein, 1987; Knierim \& Van Essen, 1992), and this could propagate to later stages of the visual system, influencing the ERP waveform for hundreds of milliseconds.

None of the published ERP studies of attention capture meet all of these criteria. In the present study, we followed these principles in a series of experiments that were designed to determine whether salient singletons capture attention in a bottom-up fashion. First, the target stimuli were not singletons along any dimension, and thus, the participants could not use singleton-detection mode to find them (Principle 1). Second, a short stimulus duration $(200 \mathrm{msec})$ was used in order to minimize the possibility that the participants would engage in a serial search of the display, and attention was cued to the region that could contain the irrelevant singleton (Principle 2). Third, irrelevant singletons were presented in the search array at a time when the participants expected that a target might be presented (Principle 3). Fourth, to rule out the possibility that the observed ERP effects were a consequence of imbalances in sensory energy, a control experiment was included, in which the effects of sensory imbalances were directly assessed (Principle 4).

In addition to taking into account these principles, we also considered the possibility that salient irrelevant singletons might be actively suppressed. Previous studies of selective attention have demonstrated that selective attention operates by a combination of facilitation and suppression (Luck, 1995; Luck et al., 1994; Munneke, Van der Stigchel, \& Theeuwes, 2008; Ruff \& Driver, 2006; Slotnick, Schwarzbach, \& Yantis, 2003). This raises the possibility that salient stimuli always trigger an attentional capture signal (an attend-to-me signal), but suppression mechanisms may prevent this signal from leading to capture. In fact, researchers using a cuing paradigm have reported that reaction times (RTs) for targets in a search display are longer when the target appears at the location of the singleton distractors in the preceding cue display (Lamy \& Egeth, 2003; Lamy, Leber, \& Egeth, 2004). Other studies show that attention capture can be reduced if the irrelevant singleton occurs on a large majority of trials (Geyer, Müller, \& Krummenacher, 2008) or if the color of the singleton is predictable (Theeuwes \& Burger, 1998). In addition, both experimental and correlational studies have shown that attention capture is stronger when the availability of working memory resources is re- duced (Barrett, Tugade, \& Engle, 2004; Fukuda \& Vogel, 2009; Lavie \& de Fockert, 2005; Vogel, McCollough, \& Machizawa, 2005). Together, these studies suggest that the ability to ignore a salient singleton is mediated by an active, top-down suppression process. However, in most of these studies, suppression of the irrelevant singleton was measured through an examination of the effects of the singleton on target-detection speed, which is indirect. That is, the speed of the response to the target was used to draw inferences about whether attention was directed to the irrelevant singleton.

To provide converging evidence, we examined the $\mathrm{Pd}$ (distractor positivity) component as an electrophysiological marker of attentional suppression. The Pd component was first described in detail by Hickey, Di Lollo, and McDonald (2009), who asked participants to discriminate the identity of a target item at one location in the presence of a single distractor item at a different location. The Pd was observed as a more positive voltage at contralateral scalp sites than at ipsilateral sites relative to the position of the distractor item. The N2pc component was also observed in this study as a contralateral negativity with respect to position of the target item. The N2pc and Pd components had similar topographies, with maximal voltage at lateral occipitotemporal electrode sites. Although the functional significance of the Pd component has not yet been studied in detail, three pieces of evidence suggest that the Pd component reflects suppression of the distractor. First, the Pd component is observed contralateral to the distractor, even when the target is not lateralized. Second, the polarity of the Pd component is opposite to that of the N2pc component. Third, the Pd component is eliminated if the participants are required only to detect the presence of the target item, rather than to discriminate its identity, which reduces the need to actively suppress the distractor. In the present study, we sought to determine whether this electrophysiological sign of active suppression would be observed in response to salient irrelevant singletons.

\section{EXPERIMENT 1}

In accord with the experimental design principles described previously, in this experiment, we used a task in which participants searched for a feature-defined nonsingleton target within a prespecified region of the display. As is shown in Figure 1, each array contained four letters in the upper field and four letters in the lower field. The participants searched for targets that were defined by a combination of letter size and identity (e.g., a large A). The to-be-attended region was cued at the beginning of each block, and the participants responded when they detected the target letter within the cued region by pushing a button (no response was to be made when the target was absent or when it was presented within the uncued region). By explicitly manipulating attention in this manner, it was possible (1) to provide direct evidence that the stimuli within the to-be-attended region were, in fact, attended; (2) to obtain converging evidence about the role of spatial attention in modulating attention capture; and (3) to determine whether suppression of the irrelevant singleton, 

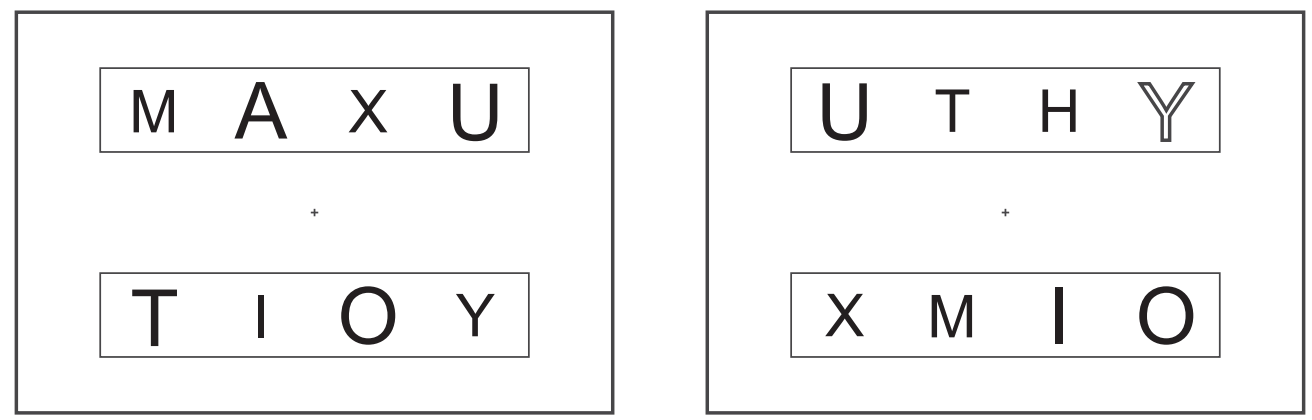

Figure 1. Example of search displays used in Experiments 1 and 2. In the target, target-similar, and standard arrays (left panel), all letters were the same color (either red or green). In the salient distractor arrays (right panel), one letter was red and the other letters were green (or vice versa). The central fixation and the two outlined rectangles were gray. All stimuli were presented against a black background.

if one is present, is modulated by spatial attention. Each array could contain the target letter, a target-similar distractor (i.e., the correct letter in the wrong size), or a salient distractor (a nontarget letter presented in a unique color), within either the upper region or the lower region. Arrays never contained both a target and a target-similar distractor or salient distractor. ${ }^{2}$ In half of the trial blocks, all of the items were red except the salient distractor, which was green. These colors were reversed for the remaining trial blocks; the red-standard and green-standard blocks occurred in random order (Experiment 4 will show that the same pattern of results is obtained when the colors reverse unpredictably from trial to trial within each block).

We predicted that targets would elicit an N2pc component, but only when appearing within the to-be-attended region, reflecting the allocation of attention to the target. We further predicted that the target-similar distractors would elicit an N2pc component when they appeared within the to-be-attended region, which would confirm that the top-down attentional set was properly directed toward the task-relevant feature and that spatial attention was focused on the appropriate region.

We anticipated three possible outcomes for the salient singleton distractor. First, if attention is deployed toward salient distractors in a completely bottom-up fashion, the salient singleton distractor should elicit an N2pc component (as has been found many times when color singletons were targets; see, e.g., Luck \& Hillyard, 1994a, 1994b). This N2pc component might be limited to singletons presented within the to-be-attended region if salient singletons capture attention only when they appear within the focus of spatial attention (as is proposed by the modified version of the bottom-up saliency hypothesis). In addition, if the salient distractor elicits an N2pc component, the duration of the $\mathrm{N} 2 \mathrm{pc}$ component may be reduced if attention is rapidly redeployed after being captured by the irrelevant singleton. However, some significant N2pc activity should be seen for the irrelevant singletons if they capture attention in a truly bottom-up manner. A second possibility is that the salient singletons will elicit no significant lateralized ERP activity, indicating the complete absence of a bottom-up attention capture signal, as would be expected on the basis of the contingent involuntary orienting hypothesis. The dimension-weighting account of Müller and colleagues would make this same prediction (Found \& Müller, 1996; Müller, Heller, \& Ziegler, 1995), because it assumes that irrelevant dimensions are given low weight in the competition for attention. A third possibility is that the salient singleton will elicit a Pd component. This would provide unambiguous evidence that the singleton triggered a distinctive signal within the visual cortex (e.g., an attend-to-me signal), and it would suggest that a topdown suppression mechanism was directed to the singleton to prevent it from actually capturing attention.

\section{Method}

Participants. The participants in all experiments reported here were neurologically normal volunteers between 18 and 30 years old who were paid for their participation. All of the participants had normal or corrected-to-normal vision. Twelve paid volunteers participated in each experiment.

Stimuli and Procedure. The stimuli were presented on a video monitor at a distance of $70 \mathrm{~cm}$ with a black background. The display contained a continuously visible gray fixation cross $\left(0.4^{\circ} \times\right.$ $\left.0.4^{\circ}, 11.55 \mathrm{~cd} / \mathrm{m}^{2}\right)$ at the center of the display and two gray rectangles $\left(18.5^{\circ}\right.$ wide $\times 3.5^{\circ}$ tall, $\left.11.55 \mathrm{~cd} / \mathrm{m}^{2}\right)$, centered $4.5^{\circ}$ above and below the fixation cross. As is illustrated in Figure 1, each stimulus array consisted of eight uppercase letters, four in the upper visual field and four in the lower visual field. The letters were selected from the set $\mathrm{A}, \mathrm{H}, \mathrm{I}, \mathrm{M}, \mathrm{O}, \mathrm{T}, \mathrm{U}, \mathrm{X}$, and $\mathrm{Y}$. In each stimulus array, two small $\left(1.6^{\circ}\right.$ wide $\times 2.0^{\circ}$ tall $)$ and two large letters $\left(2.0^{\circ} \times 2.5^{\circ}\right)$ were placed inside each upper and lower rectangle. Each letter was centered $4.5^{\circ}$ above or below the horizontal meridian and $5^{\circ}$ or $8^{\circ}$ to the left or right of the vertical meridian. The letter identity and the letter size at each stimulus location were varied randomly across trials, with the constraints that no identity repeated within a presentation and that the number of large and small letters within each rectangle was equal. Stimulus duration was $200 \mathrm{msec}$, and the interval between successive stimulus onsets varied randomly between 800 and $900 \mathrm{msec}$. Each participant performed 100-150 practice trials, followed by 72 blocks of 56 trials during which ERPs were recorded.

At the beginning of each block, one of the eight letter identities and its size (small or large) was designated as the target (e.g., a large A) and either the upper or the lower visual field was designated as the attended area for that block. The participants were instructed to direct their attention to the instructed visual field while maintaining fixation on the fixation point and to respond when they detected the target letter within the attended area by pushing a button with their right thumb. They were instructed not to respond to target letters within the unattended area. Speed and accuracy were equally 
stressed. Trials in which RTs were shorter than $100 \mathrm{msec}$ or longer than $800 \mathrm{msec}$ were excluded from all analyses (averages of $1.6 \%$ and $1.1 \%$ of correct target trials, respectively).

Seven types of stimulus arrays were presented, each with equal probability (576 trials, approximately $14.3 \%$ ): target within the attended area, target within the unattended area, ${ }^{3}$ target-similar distractor within the attended area, target-similar distractor within the unattended area, salient distractor within the attended area, salient distractor within the unattended area, and standard (i.e., trials without a target, target-similar distractor, or salient distractor). The target-similar distractor had the same letter identity as the target but a different letter size (e.g., if the target was a large A, the target-similar distractor was a small A). The salient distractor had a different color from the other letters and had a randomly chosen letter identity and size, except that it was never the same letter as the target. In half of the trial blocks, all of the items were red $\left(u^{\prime}=\right.$ $\left..45, v^{\prime}=.51 ; 19.84 \mathrm{~cd} / \mathrm{m}^{2}\right)$, except the salient distractor, which was green $\left(u^{\prime}=.14, v^{\prime}=.55 ; 25.76 \mathrm{~cd} / \mathrm{m}^{2}\right)$. These colors were reversed for the remaining trial blocks. The red-standard and green-standard blocks occurred in random order.

Recording and Analysis. The EEG was recorded using active $\mathrm{Ag} / \mathrm{AgCl}$ electrodes (BioSemi ActiveTwo system) from the left and right mastoids and 32 scalp sites (Fp1, Fp2, F7, F3, Fz, F4, F8, T7, C3, Cz, C4, T8, P9, P7, P5, P3, P1, Pz, P2, P4, P6, P8, P10, PO7, $\mathrm{PO} 3, \mathrm{POz}, \mathrm{PO} 4, \mathrm{PO} 8, \mathrm{O} 1, \mathrm{Oz}, \mathrm{O} 2$, and Iz, according to the modified 10-20 system; American Electroencephalographic Society, 1994). To detect eye movements and blinks, the electrooculogram (EOG) was recorded from electrodes placed at the outer canthi of each eye and above and below the right eye. All signals were recorded in single-ended mode and referenced offline. The EEG and EOG were low-pass filtered with a 5th-order sinc filter (half-amplitude cutoff at $205 \mathrm{~Hz}$ ) and digitized at $1024 \mathrm{~Hz}$.

All analyses after data acquisition were conducted using ERPLAB Toolbox (www.erpinfo.org/erplab/) and EEGLAB Toolbox (Delorme \& Makeig, 2004; http://sccn.ucsd.edu/eeglab/), which are freely available, open source, MATLAB-based packages for EEG/ ERP data analysis. The EEG signals were rereferenced to the average of the left and right mastoids, and the four EOG signals were rereferenced into bipolar vertical and horizontal EOG derivations. These signals were low-pass filtered offline using a noncausal Butterworth infinite impulse response filter with a half-amplitude cutoff at $30 \mathrm{~Hz}$ and a roll-off of $12 \mathrm{~dB} /$ octave and then down-sampled to $256 \mathrm{~Hz}$. Averaged ERP waveforms were computed with a 500msec epoch, beginning $100 \mathrm{msec}$ before stimulus onset. Trials were automatically excluded if they contained an incorrect response (i.e., no response for target trials or any response for nontarget trials), if the RTs for targets were shorter than $100 \mathrm{msec}$ or longer than $800 \mathrm{msec}$, if the EEG exceeded $\pm 100 \mu \mathrm{V}$ in any channel, or if the EOG exceeded $\pm 80 \mu \mathrm{V}$. To assess residual eye movements, we computed averaged horizontal EOG waveforms for left- and rightstimulus trials. We replaced any participants for whom the residual EOG activity was more than $3.2 \mu \mathrm{V}$, which means that the residual eye movements in the remaining participants were less than $0.2^{\circ}$ with propagated voltage of less than $0.1 \mu \mathrm{V}$ at the posterior scalp sites (Lins, Picton, Berg, \& Scherg, 1993). We also replaced participants for whom more than $25 \%$ of trials were rejected because of EEG/EOG artifacts. Two participants were replaced for these reasons in the present experiment. Among the final set of 12 participants, artifacts led to the rejection of an average of $7.9 \%$ of trials (range $=0.3 \%-21.3 \%)$.

The N2pc and Pd components were measured from contralateralminus-ipsilateral difference waves at the PO7 and PO8 electrode sites as the mean voltage between 225 and $300 \mathrm{msec}(\mathrm{N} 2 \mathrm{pc})$ and between 115 and $225 \mathrm{msec}(\mathrm{Pd})$ relative to the mean voltage during the $100-\mathrm{msec}$ prestimulus baseline period. All ANOVAs used Greenhouse-Geisser corrections to the degrees of freedom, and only the corrected probability values are reported. The waveforms were collapsed across letter identities, sizes, colors, and locations before the component amplitudes were measured to eliminate sensory confounds related to these factors.

\section{Results and Discussion}

Behavior. The mean RT for targets was $514 \mathrm{msec}$, and the mean hit rate was $85.5 \%$. The mean false-positive rate was $2.1 \%$ for targets within the unattended area, $9.3 \%$ for target-similar distractors within the attended area, 1.8\% for target-similar distractors within the unattended area, $1.5 \%$ for salient distractors within the attended area, $1.5 \%$ for salient distractors within the unattended area, and 1.6\% for standards. A one-way ANOVA performed on the mean false-positive rates revealed a main effect of stimulus category $[F(1,11)=35.7, p<.001]$, and follow-up $t$ tests between the standards and each other type of nontarget indicated that only the target-similar distractors within the attended area showed a higher false-positive rate than the standards $(p<.001)$. The finding of an increased false alarm rate only for the target-similar distractors within the attended area indicates that the manipulation of spatial attention was effective and that the target-similar distractors were indeed quite similar to the targets. Further evidence of the effectiveness of the spatial attention manipulation will be provided in Experiment 2, in which participants were required to divide their attention across the top and bottom sets of letters, which led to lower target detection performance.

Electrophysiology. Figure 2 shows the ERP waveforms from lateral occipital scalp sites (PO7 and PO8) for targets, target-similar distractors, and salient distractors, averaged across participants. Separate waveforms are shown for contralateral and ipsilateral sites, relative to the item of interest (e.g., the contralateral waveform for the salient distractor was the average of the lefthemisphere electrode when the salient distractor was in the right visual field and the right-hemisphere electrode when the salient distractor was in the left visual field; the ipsilateral waveform for the salient distractor was the average of the left-hemisphere electrode when the salient distractor was in the left visual field and the righthemisphere electrode when the salient distractor was in the right visual field). When the targets were presented within the attended area, the N2pc component was present as a negative deflection in the ERP waveform at contralateral relative to ipsilateral scalp sites, beginning at approximately $200 \mathrm{msec}$ poststimulus. The N2pc component was also elicited in response to target-similar distractors within the attended area, although it was slightly smaller than the N2pc component for the targets. However, no N2pc activity was present for either the targets or the target-similar distractors within the unattended area. The Pd component was observed for salient distractors as a positivity at contralateral relative to ipsilateral electrode sites, and this effect was approximately equivalent whether the salient distractor was presented within the attended area or the unattended area. Because the Pd component was not observed for target-similar distractors, we can be certain that the Pd effect to salient distractors was associated with the processing of salient 


\section{Attended Area}

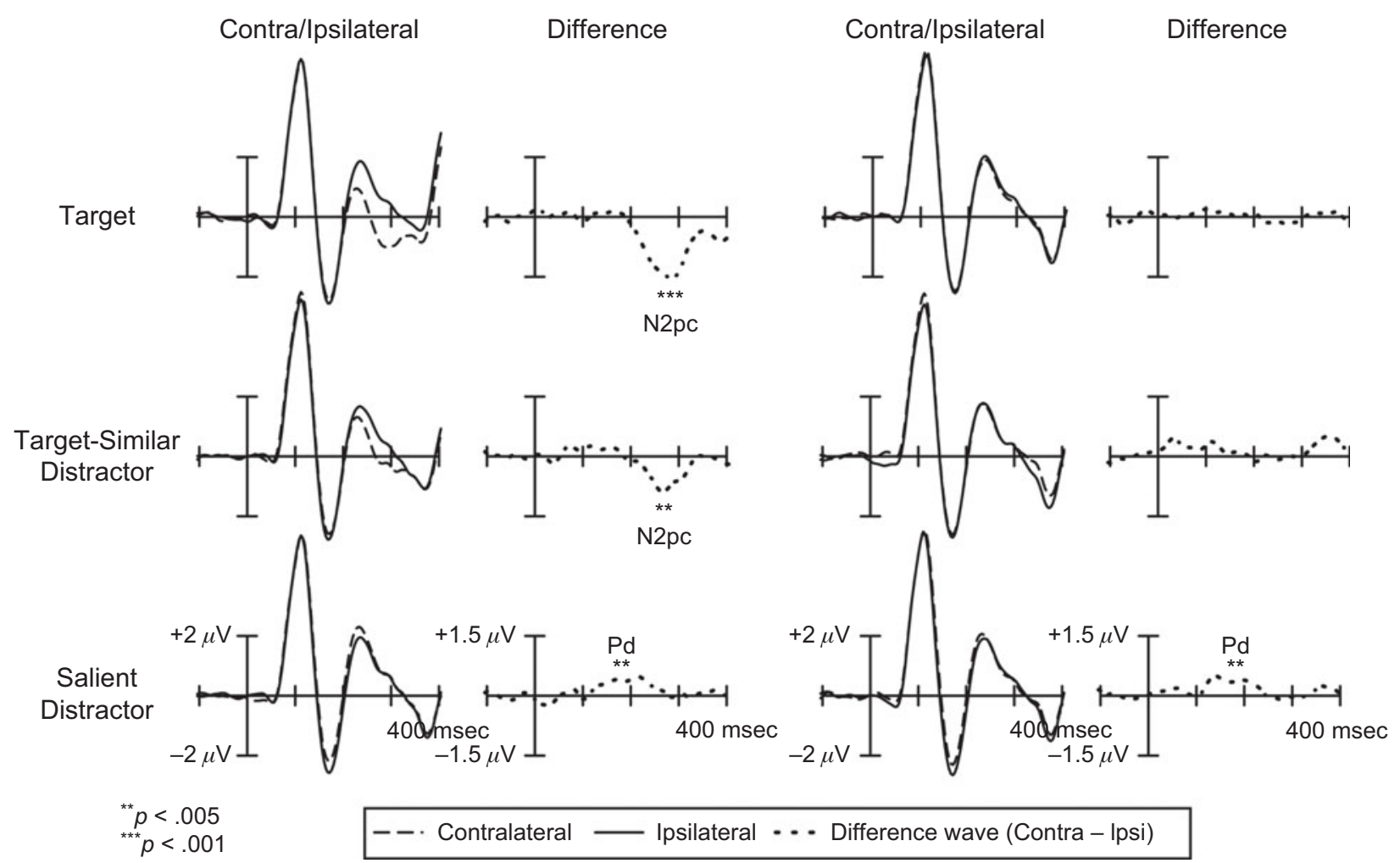

Figure 2. Grand average waveforms from Experiment 1 for each stimulus category at contralateral versus ipsilateral PO7/PO8 electrode sites, along with the difference between the contralateral and ipsilateral waveforms (which isolates the N2pc and Pd components).

distractors, rather than with some general response to nontarget stimuli.

The Pd effect for salient distractors began at approximately $100 \mathrm{msec}$, whereas the N2pc effect for targets and target-similar distractors began at approximately $200 \mathrm{msec}$. The earlier onset latency of the Pd relative to the N2pc component presumably reflects the greater salience of the salient distractor than of the target (see Luck et al., 2006, for evidence that N2pc onset time varies with salience). Because of this latency difference, we used separate time windows to focus on these components (115-225 msec for Pd; 225-300 msec for N2pc). These windows were chosen because they consistently captured the Pd and N2pc effects across a number of experiments (including the present experiments and additional experiments that yielded converging results). Note, however, that some N2pc activity was present in the Pd time window, and some Pd activity was present in the N2pc time window, so these windows do not completely isolate the two components.

$\mathrm{N} 2 \mathrm{pc}$ amplitude from the contralateral-minusipsilateral difference waves was entered into a two-way ANOVA with factors of stimulus category (target, targetsimilar distractor, salient distractor) and spatial attention (attended area, unattended area). This ANOVA revealed significant main effects of stimulus category $[F(1,11)=$
19.7, $p<.001]$ and spatial attention $[F(1,11)=24.1$, $p<.001$ ], along with a significant interaction between these factors $[F(1,11)=19.2, p<.001]$. This interaction was decomposed with planned comparisons, which revealed that the mean amplitude within the attended area was significantly larger for targets than for either the target-similar distractors $(p<.025)$ or the salient distractors $(p<.001)$. However, there were no significant differences between these stimulus categories within the unattended area. Furthermore, the mean amplitude of targets and target-similar distractors was larger within the attended area than within the unattended area $(p$ s $<.001$ for both targets and target-similar distractors), whereas there was no such difference for the salient distractors. One-sample $t$ tests versus zero revealed that a significant $\mathrm{N} 2 \mathrm{pc}$ component was generated for targets within the attended area $[t(11)=-6.5, p<.001]$ and for targetsimilar distractors within the attended area $[t(11)=$ $-4.0, p<.005]$, whereas the N2pc component did not significantly differ from zero for targets within the unattended area $[t(11)=-1.3]$, for target-similar distractors within the unattended area $[t(11)=-0.3]$ or for salient distractors within either the attended area $[t(11)=1.3]$ or the unattended area $[t(11)=-0.3]$.

These N2pc results confirmed that spatial attention was directed toward the to-be-attended area and that the par- 
ticipants searched items possessing the target letter identity within that area. These findings are consistent with the behavioral results, in which the target-similar distractors showed a higher false-positive rate only when they were presented within the attended area. The lack of an $\mathrm{N} 2 \mathrm{pc}$ component for the salient distractors demonstrates that the mechanism of attention reflected by the N2pc component is not present for salient distractors under conditions that provide no incentive to search for singletons and that instead emphasize feature-based target selection. This finding further demonstrates that the lack of effects of salient singleton distractors in many previous behavioral experiments (e.g., Bacon \& Egeth, 1994; Folk et al., 1992; Leber \& Egeth, 2006) cannot be explained by positing that attention is captured by these singletons without a measureable impact on target-detection performance (e.g., by a very brief capture of attention, as was proposed by Theeuwes, Atchley, \& Kramer, 2000).

The Pd component was analyzed just like the N2pc component, except with a measurement window of 115$225 \mathrm{msec}$. The ANOVA yielded a significant main effect of stimulus category $[F(1,11)=7.6, p<.005]$ but no significant main effect of spatial attention and no significant interaction between stimulus category and spatial attention. Follow-up tests indicated that Pd amplitude was significantly greater for the salient distractors than for the targets $(p<.02)$ or for the target-similar distractors $(p<$ $.05)$. One-sample $t$ tests versus zero revealed that a significant Pd component was generated for the salient distractors within both the attended area $[t(11)=4.0, p<.005]$ and the unattended area $[t(11)=3.8, p<.005]$, but no significant Pd component was generated for targets or for target-similar distractors within either area.

Because we observed no N2pc evidence that attention is deployed to salient distractors even within the attended area, the present data do not support the bottom-up saliency hypothesis, which posits that attention always first shifts to the most salient item in the visual field, irrespective of the participant's goal (Belopolsky et al., 2007; Hickey et al., 2006; Theeuwes, 1991a, 1992, 2004). Thus, when precautions are taken to ensure that the task set does not encourage the participants to employ singletondetection mode, salient singletons do not inevitably capture the mechanism of attention indexed by the N2pc component.

Instead, the finding of a putative ERP index of distractor suppression $(\mathrm{Pd})$ suggests that attentional suppression was generated in response to salient distractors. This active suppression was observed for singletons within both the unattended and attended areas, indicating that salient distractors generate a bottom-up capture signal even when attention is focused elsewhere. Thus, these results demonstrate that, consistent with the bottom-up saliency hypothesis, salient singletons are detected and generate a location-specific signal. However, they are also consistent with the contingent involuntary orienting hypothesis insofar as this signal did not lead to the allocation of attention. Thus, these results support a hybrid theory, in which salient singletons generate a signal that ordinarily leads to the capture of attention but can be suppressed on the basis of top-down attentional control settings.

Importantly, the lack of a significant difference in the Pd component between the attended and unattended areas implies that eye positions did not move from the central fixation to the to-be-attended area at the beginning of the block (or that this effect is not influenced by eye position). In addition, to minimize this possibility, a short block length (48 sec) was used, and the experimenter closely monitored the vertical EOG signal and alerted the subject if eye movements were observed.

Note that the sensory imbalance present in arrays containing a salient singleton could have been responsible for the lateralized ERP effects. This possibility will be ruled out in Experiment 3. Also note that in at least a dozen previous experiments, it has been found that color singletons such as those used in this experiment will elicit an N2pc component rather than a Pd component if they are targets (Eimer, 1996; Eimer \& Kiss, 2008; Hilimire, Mounts, Parks, \& Corballis, 2009; Luck, Fan, \& Hillyard, 1993; Luck \& Ford, 1998; Luck, Girelli, McDermot, \& Ford, 1997; Luck \& Hillyard, 1994a, 1994b; Mazza, Turatto, \& Caramazza, 2009; Mazza, Turatto, Umiltà, \& Eimer, 2007; Schubö \& Müller, 2009). This makes the finding of a Pd component here even more remarkable.

The N2pc component for targets and target-similar distractors was not observed when they appeared within the unattended area, suggesting that the attentional deployment toward items with task-relevant features is strongly restricted by spatial attention. However, results from several previous studies have led to the conclusion that spatial attention cannot prevent contingent attentional capture (Folk, Leber, \& Egeth, 2002; Seiss, Kiss, \& Eimer, 2009; Theeuwes, Kramer, \& Atchley, 2001). The difference in stimulus salience may account for these discrepant results. That is, both the targets and the distractors in the previous studies were salient singletons, whereas the targets and target-similar distractors in the present study were not salient. Thus, this suggests that if participants attend to salient targets, salient distractors may capture attention regardless of spatial attention. However, if the participants attend to nonsalient targets, nonsalient distractors within the unattended area can be ignored even if they contain task-relevant features.

\section{EXPERIMENT 2}

In Experiment 1, either the upper or lower visual field was attended, and the participants searched for targets within the attended area. However, in most previous studies, attentional capture was investigated in the situations in which participants were searching for targets throughout the array. Although we found that salient distractors elicited a Pd component, it is possible that this effect is limited to situations in which a particular area is attended and other areas are ignored. Experiment 2 was conducted to further investigate whether the distractor suppression process indexed by $\mathrm{Pd}$ is also observed when spatial attention is not limited to one portion of the array. This experi- 
ment also served to assess the replicability of the pattern of results observed in Experiment 1.

\section{Method}

The stimuli and procedure in this experiment were identical to those used in Experiment 1, except as follows. The participants were required to attend throughout the array and respond when they detected the target, regardless of whether it appeared in the upper or lower visual field. Four types of stimulus arrays were presented: target (576 trials, approximately $28.6 \%$ ), target-similar distractor (576 trials, approximately $28.6 \%$ ), salient distractor ( 576 trials, approximately $28.6 \%$ ), and standard (288 trials, approximately $14.3 \%$ ). Averages of $4.4 \%$ and $1.8 \%$ of correct target trials were excluded from all analyses because their RTs were shorter than $100 \mathrm{msec}$ or longer than $800 \mathrm{msec}$, respectively. Each participant performed $100-150$ practice trials, followed by 36 blocks of 56 trials during which ERPs were recorded. No participants were replaced owing to residual EOG or EEG artifacts. An average of $9.1 \%$ of trials were rejected because of artifacts (range $=2.7 \%-22.9 \%$ ).

\section{Results and Discussion}

Behavior. The mean RT for targets was $534 \mathrm{msec}$, and the mean hit rate was $71.6 \%$. Target detection performance in this experiment was significantly less accurate than that in Experiment $1[t(22)=-4.5, p<.001]$, providing additional evidence that the participants were able to focus processing resources on the attended region in Experiment 1 . The RTs were also longer in the present experiment, but the difference between experiments did not reach significance $[t(22)=1.3]$. The mean false-positive rate in Experiment 2 was $14.8 \%$ for target-similar distractors, $5.0 \%$ for salient distractors, and $4.7 \%$ for standards. A one-way ANOVA performed on the false-positive rates revealed a main effect of stimulus category $[F(1,11)=24.1$, $p<.001]$, and follow-up $t$ tests indicated that only the target-similar distractors exhibited a significantly higher false-positive rate than did the standards $(p<.001)$.

Electrophysiology. Figure 3 shows the ERP waveforms from lateral occipital scalp sites (PO7 and PO8) for targets, target-similar distractors, and salient distractors, averaged across participants. As with the results from the attended area in Experiment 1, the N2pc component was observed for the targets and the target-similar distractors but not for the salient distractors, whereas the Pd component was observed for the salient distractors.

For the analysis of the N2pc time window (225$300 \mathrm{msec}$ ), a one-way ANOVA yielded a significant main effect of stimulus category $[F(1,11)=30.4, p<.001]$. Follow-up analyses indicated that N2pc amplitude was significantly greater for the targets than for the target-similar distractors $(p<.01)$ or the salient singleton distractors $(p<.001)$. One-sample $t$ tests versus zero revealed that a significant $\mathrm{N} 2 \mathrm{pc}$ component was generated for the targets $[t(11)=-5.9, p<.001]$ and the target-similar distractors $[t(11)=-6.4, p<.001]$ but not for the salient singleton distractors $[t(11)=1.9]$.

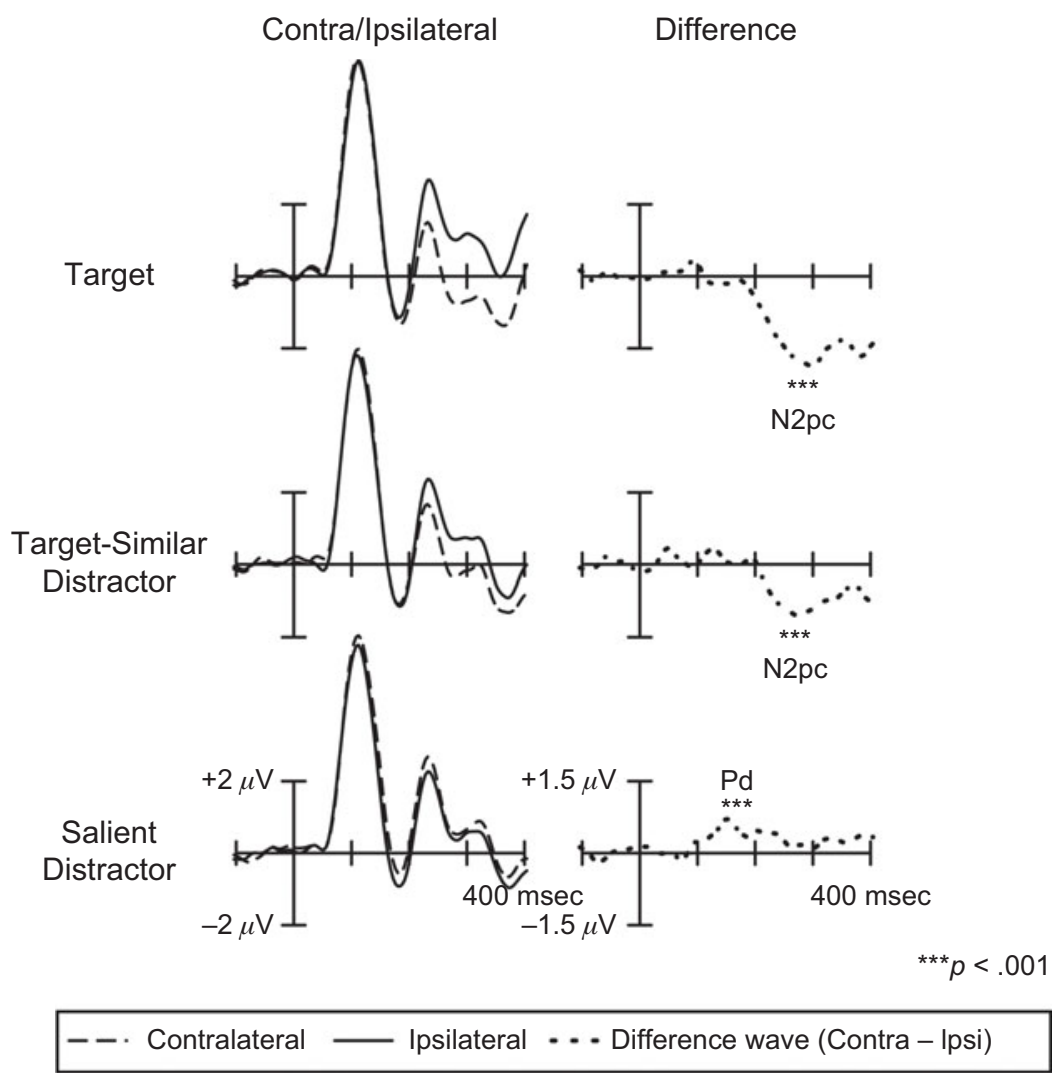

Figure 3. Grand average waveforms from Experiment 2 for each stimulus category at $\mathrm{PO} 7 / \mathrm{PO}$ and the difference between contralateral and ipsilateral waveforms. 

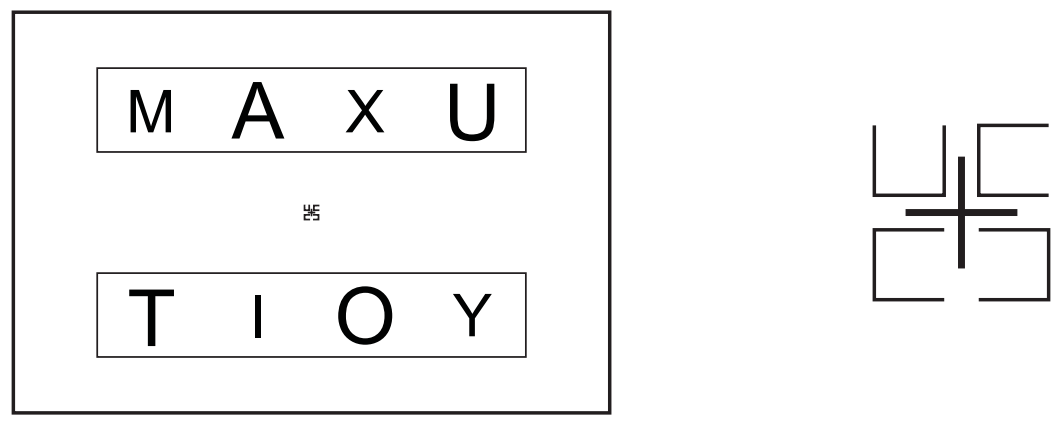

Figure 4. Example of search displays used in Experiment 3 (left panel) and square stimuli around fixation in close up (right panel). All squares were gray, three had a gap on their right or left sides, but one had a gap on its upper or lower side.

For the analysis of the Pd time window (115-225 msec), a one-way ANOVA yielded a significant main effect of stimulus category $[F(1,11)=18.6, p<.001]$. Followup analyses indicated that $\mathrm{Pd}$ amplitude was significantly greater for the salient distractors than for the target $(p<$ $.001)$ or for the target-similar distractors $(p<.03)$. Onesample $t$ tests versus zero revealed that a significant Pd component was generated only for the salient distractors $[t(11)=5.2, p<.001]$.

These results replicate and extend those of Experiment 1 , demonstrating once again that salient nontarget singletons do not inevitably capture attention, and that, instead, they may be actively suppressed.

\section{EXPERIMENT 3}

In both Experiments 1 and 2, the Pd component was elicited by salient distractors. Because the salient distractors were always lateralized stimuli, it is possible that the Pd effect may actually reflect a sensory response caused by the low-level sensory difference between the right and left visual fields, rather than reflecting an active, top-down suppression of the singleton. Experiment 3 was designed to rule out this possibility by demonstrating that the same irrelevant singletons used in Experiments 1 and 2 will fail to elicit a lateralized response when the task is changed.

In Experiment 3, four small squares were presented around central fixation in addition to the surrounding letter stimuli in the preceding experiments, and participants performed a demanding visual search task with these central stimuli, ignoring the more peripheral letters (see Figure 4). This particular search has been shown to involve the serial application of attention (Woodman \& Luck, 1999, 2003), and we reasoned that attention would be so focused and held at the central region by this highly perceptually demanding task that it would not be necessary to further suppress salient singletons at peripheral locations. That is, when the perceptual load is sufficiently high, salient stimuli are less likely to attract attention (Cosman \& Vecera, 2009; Lu \& Han, 2009), so active suppression may not be necessary when the load is sufficiently high. In addition, suppression of the salient irrelevant singletons in Experiments 1 and 2 may have been necessary to prevent them from eliciting an eye movement, but this may not be necessary when the observers know that the targets will always be presented near fixation. Thus, if the Pd component in Experiments 1 and 2 reflected the active suppression of the salient singleton distractors, it should be absent in the present experiment. If, however, the Pd component in Experiments 1 and 2 was simply a low-level sensory response reflecting imbalances in sensory energy between the right and left visual fields, it should again be observed in the present experiment.

\section{Method}

The stimuli and procedure were identical to those used in the preceding experiments, except as follows. Each array again contained eight letters, but they were accompanied by four gray squares $\left(0.25^{\circ} \times 0.25^{\circ}, 11.55 \mathrm{~cd} / \mathrm{m}^{2}\right)$, each centered $0.25^{\circ}$ diagonally from the fixation point (Figure 4, left panel). Three squares had a gap $\left(0.25^{\circ}\right)$ on the right or left side, randomly determined, and one randomly selected square had a gap on the top or bottom (Figure 4 right panel). At the beginning of each block, the participants were told that either the top gap or the bottom gap would be the target for that block. A target was presented on $50 \%$ of the trials, and the participants pressed a button with the right thumb when the target was detected. The letter arrays were constructed just as in the preceding experiments, but they were completely task irrelevant. A salient color singleton was again present in the letter array on $28.6 \%$ of trials. Averages of $3.3 \%$ and $1.0 \%$ of correct target trials were excluded from all analyses because their RTs were shorter than $100 \mathrm{msec}$ or longer than $800 \mathrm{msec}$, respectively.

The EEG recording and analysis were identical to those used in the preceding experiments, except as follows. The waveforms were collapsed across gap types and locations, as well as letter identities, sizes, colors, and locations before component amplitudes were measured, to eliminate sensory confounds related to these factors. One participant was replaced because of EEG artifacts (i.e., more than $25 \%$ of the trials were rejected because of artifacts). Among the final set of 12 participants, artifacts led to the rejection of an average of $10.2 \%$ of trials (range $=1.1 \%-21.7 \%$ ).

\section{Results and Discussion}

Behavior. When the salient distractor was absent from the letter array, the mean RT for target-present central arrays was $493 \mathrm{msec}$, the mean hit rate for target-present central arrays was $79.8 \%$, and the mean false-positive rate for target-absent central arrays was $17.8 \%$. When the salient distractor was present in the letter array, the mean RT for target-present central arrays was $492 \mathrm{msec}$, the mean hit rate for target-present central arrays was $80.8 \%$, 


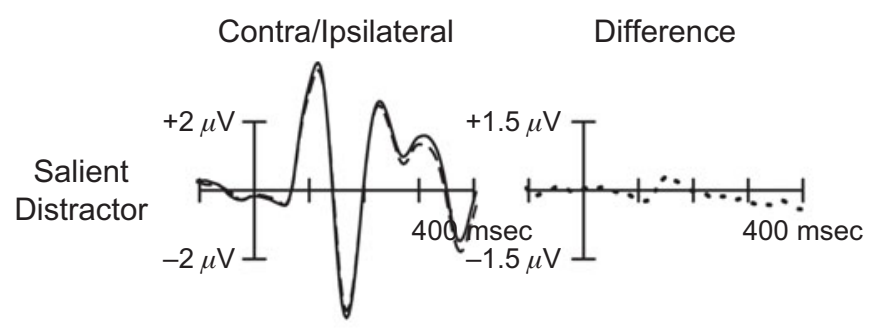

$$
\text { - - Contralateral — Ipsilateral } \cdots \text { Difference wave (Contra - Ipsi) }
$$

Figure 5. Grand average waveforms from Experiment 3 for salient distractors at $\mathrm{PO} 7 / \mathrm{PO} 8$ and the difference between contralateral and ipsilateral waveforms.

and the mean false-positive rate for target-absent central arrays was $17.9 \%$. None of these small differences was significant $[t(11)<1$ in each case $]$. Thus, the participants successfully ignored the peripheral letter arrays and focused on the task-relevant central squares.

Electrophysiology. Figure 5 shows the grand average ERP waveforms from lateral occipital scalp sites (PO7 and PO8) for stimuli that contained a salient singleton in the letter array. In contrast to those in Experiments 1 and 2, the Pd component was not observed for these salient singletons.

For the analysis of the Pd time window (115-225 msec), a one-sample $t$ test versus zero revealed that no Pd component was generated for the salient distractors $[t(11)=$ $0.5]$. To demonstrate that the difference between Experiment 3 and Experiments 1 and 2 was reliable, a series of independent-samples $t$ tests was conducted on Pd amplitude for arrays containing an irrelevant singleton. Pd amplitude was significantly reduced for irrelevant singletons in Experiment 3 compared with (1) the irrelevant singletons in the attended region in Experiment $1[t(22)=2.2$, $p<.045]$, (2) the irrelevant singletons in the unattended region in Experiment $1[t(22)=2.1, p<.045]$, and (3) the irrelevant singletons in Experiment $2[t(22)=3.0, p<$ $.01]$. Thus, the Pd effect observed for irrelevant singletons in this study depends on the task and is not a low-level sensory response.

It is possible, however, that the absence of a significant Pd effect in the present experiment reflects an interaction between attention and sensory processing. That is, focusing attention onto the central stimuli may have reduced the sensory response to the peripheral stimuli, thus decreasing the magnitude of any lateralized sensory activity. However, previous studies have shown that sensory ERPs are still quite lateralized for unattended stimuli (e.g., Heinze \& Mangun, 1995; Johannes, Münte, Heinze, \& Mangun, 1995; Mangun \& Hillyard, 1988). It is therefore unlikely that the reduced Pd component in the present experiment can be explained by attentional reduction of lateralized sensory activity. Moreover, even if this turns out to be the correct explanation, the observed Pd component is still not a purely bottom-up sensory effect, because it can be modulated by top-down attention signals.

\section{EXPERIMENT 4}

In Experiments 1 and 2, the colors of the targets and the salient distractors were blocked (i.e., if the targets were red, the salient distractors were green in the red-standard trial blocks or vice versa in the green-standard trial blocks). Therefore, it is possible that the participants might have had an incentive to attend to a particular color. This may have led the irrelevant singleton to be suppressed because it was not presented in the target color rather than because it was an irrelevant singleton, per se. In addition, more items of the target color were on the nonsingleton side of the display than on the singleton side of the display, which may have led to an N2pc component to the nonsingleton side (which would lead to the same pattern of voltage as a Pd component to the singleton). Experiment 4 was conducted to rule out these possibilities by demonstrating that the salient distractors will elicit a Pd component even when the red- and green-standard trials are randomly intermixed within trial blocks and the color of the target is therefore unpredictable.

\section{Method}

The stimuli and procedure in this experiment were identical to those used in Experiment 2, except as follows. In half of the trials, all of the items were red $\left(u^{\prime}=.45, v^{\prime}=.51 ; 19.84 \mathrm{~cd} / \mathrm{m}^{2}\right)$, except the salient distractor, which was green $\left(u^{\prime}=.14, v^{\prime}=.55 ; 25.76 \mathrm{~cd} / \mathrm{m}^{2}\right)$. These colors were reversed for the remaining trials. The red-standard trials were randomly intermixed with the green-standard trials within trial blocks. Averages of $2.2 \%$ and $1.0 \%$ of correct target trials were excluded from all analyses, because their RTs were shorter than $100 \mathrm{msec}$ or longer than 800 msec, respectively. Each participant performed 100-150 practice trials, followed by 36 blocks of 56 trials during which ERPs were recorded. One participant was replaced because of EEG artifacts (i.e., more than $25 \%$ of the trials were rejected because of artifacts). Among the final set of 12 participants, artifacts led to the rejection of an average of $11.4 \%$ of trials (range $=1.0 \%-24.2 \%$ ).

\section{Results and Discussion}

Behavior. The mean RT for targets was $503 \mathrm{msec}$, and the mean hit rate was $79.3 \%$. The mean false-positive rates were $17.0 \%$ for target-similar distractors, $2.8 \%$ for salient distractors, and $2.8 \%$ for standards. A one-way ANOVA performed on the false-positive rates revealed a main effect of stimulus category $[F(1,11)=24.1, p<.001]$, and 


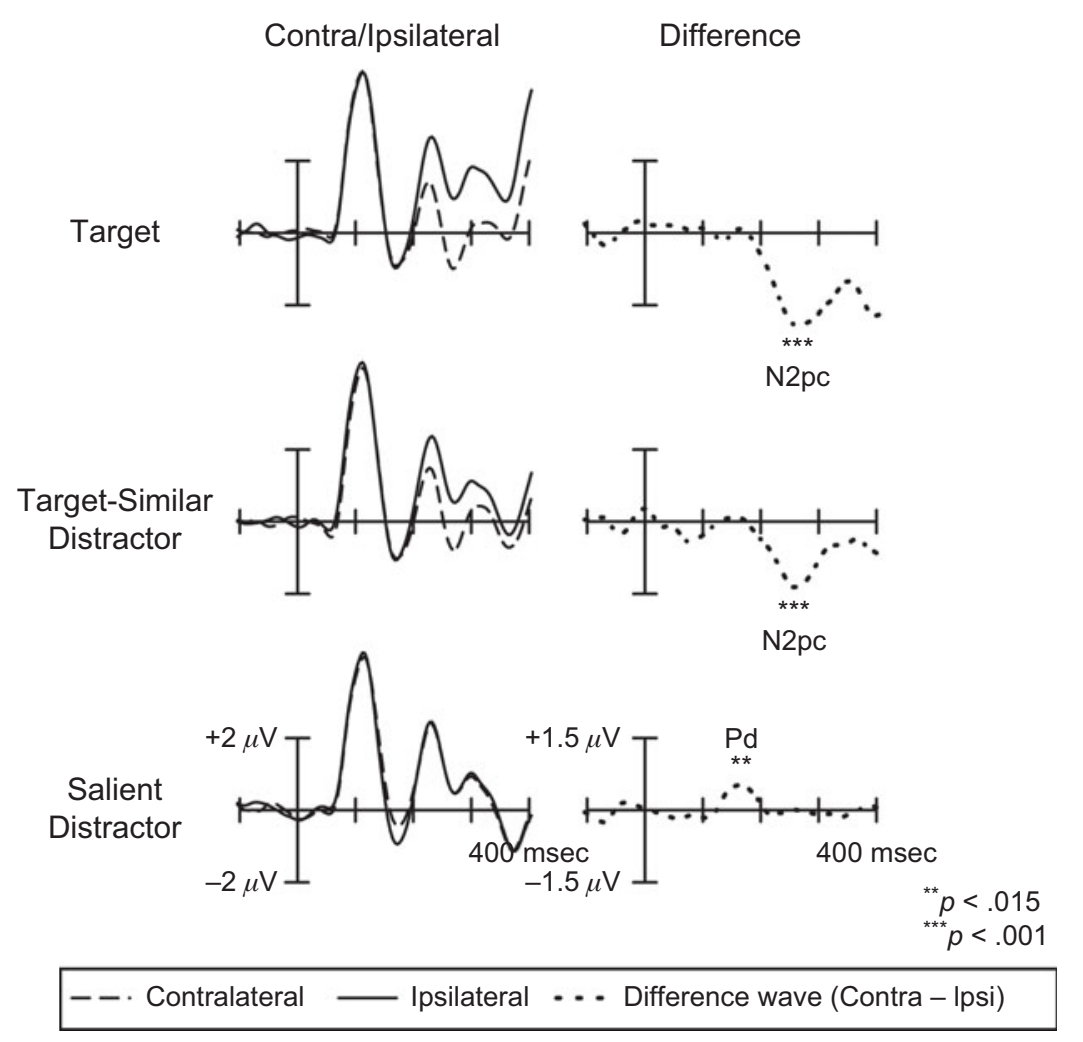

Figure 6. Grand average waveforms from Experiment 4 for each stimulus category at PO7/PO8 and the difference between contralateral and ipsilateral waveforms.

follow-up $t$ tests indicated that only the target-similar distractors exhibited a significantly higher false-positive rate than did the standards $(p<.001)$.

Electrophysiology. Figure 6 shows the ERP waveforms from lateral occipital scalp sites (PO7 and PO8) for targets, target-similar distractors, and salient distractors, averaged across participants. As with the results from the preceding experiments, the N2pc component was observed for the targets and the target-similar distractors but not for the salient distractors, whereas the Pd component was observed for the salient distractors. Topographic maps of the N2pc and Pd components are plotted in Figure 7.

For the analysis of the N2pc time window (225$300 \mathrm{msec}$ ), a one-way ANOVA yielded a significant main effect of stimulus category $[F(1,11)=40.9, p<.001]$. Follow-up analyses indicated that N2pc amplitude was significantly greater for the targets than for the target-similar distractors $(p<.005)$ or the salient singleton distractors $(p<.001)$. One-sample $t$ tests versus zero revealed that a significant $\mathrm{N} 2 \mathrm{pc}$ component was generated for the targets $[t(11)=-7.4, p<.001]$ and the target-similar distractors $[t(11)=-5.2, p<.001]$, but not for the salient singleton distractors $[t(11)=-0.3]$.

For the analysis of the Pd time window (115-225 msec), a one-way ANOVA yielded a significant main effect of stimulus category $[F(1,11)=8.6, p<.005]$. Follow-up analyses indicated that $\mathrm{Pd}$ amplitude was significantly greater for the salient distractors than for the target $(p<$ $.005)$ or for the target-similar distractors $(p<.035)$.
One-sample $t$ tests versus zero revealed that a significant $\mathrm{Pd}$ component was generated only for the salient distractors $[t(11)=3.1, p<.015]$.

These results demonstrated that the Pd effect is not associated with selective attention to a particular color to detect targets or salient distractors.

\section{GENERAL DISCUSSION}

The nature of attentional capture by salient singletons is a controversial issue. The bottom-up saliency hypothesis proposes that salient singletons capture attention solely by means of bottom-up salience, whereas the contingent involuntary orienting hypothesis proposes that capture is contingent on top-down control settings. The debate between these theories has not been settled, and the field has reached something of an impasse. The results of the present study provide a new perspective on this longstanding controversy.

First, these results provide solid evidence that the attentional mechanism reflected by the $\mathrm{N} 2 \mathrm{pc}$ component is not allocated on the basis of pure bottom-up salience. Specifically, a highly salient singleton distractor was presented within the attended region but did not elicit an N2pc component. This was not merely a null result, however, because the voltage was actually significantly reversed (creating the Pd effect). Thus, the present study provides positive evidence that the singleton distractor was salient and yet failed to elicit an N2pc component. 
Target
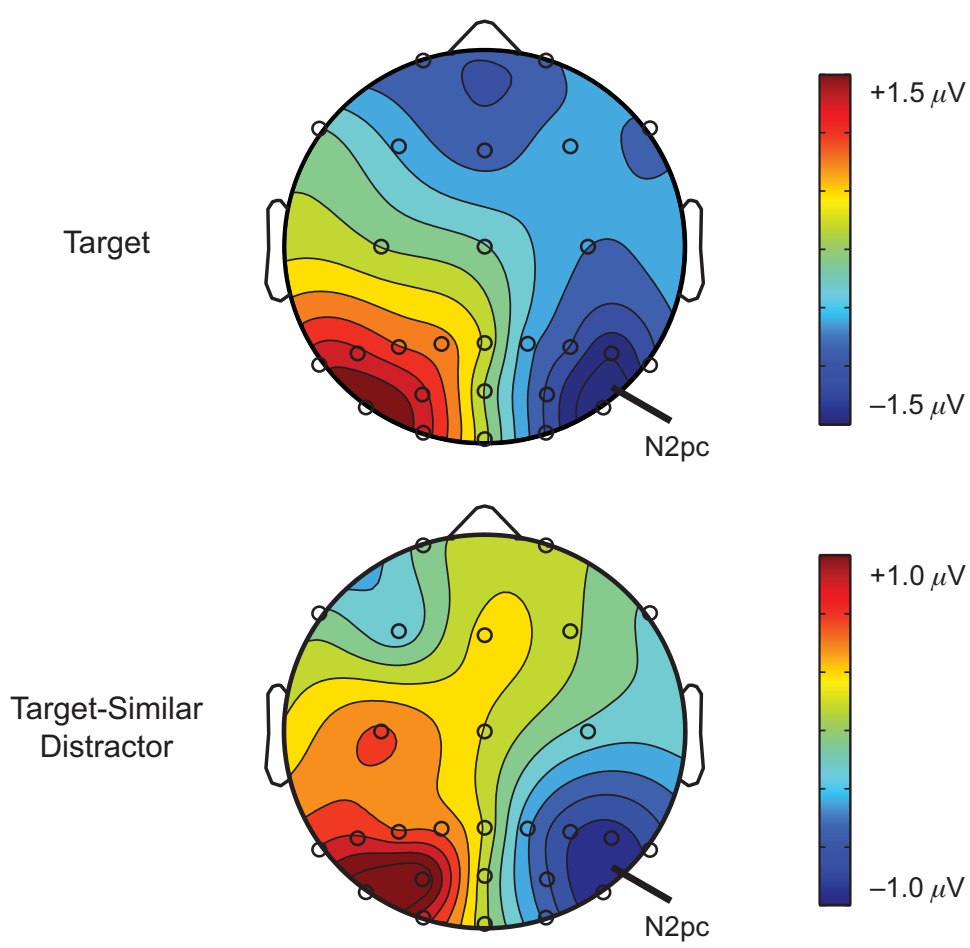

Target-Similar Distractor

Salient Distractor
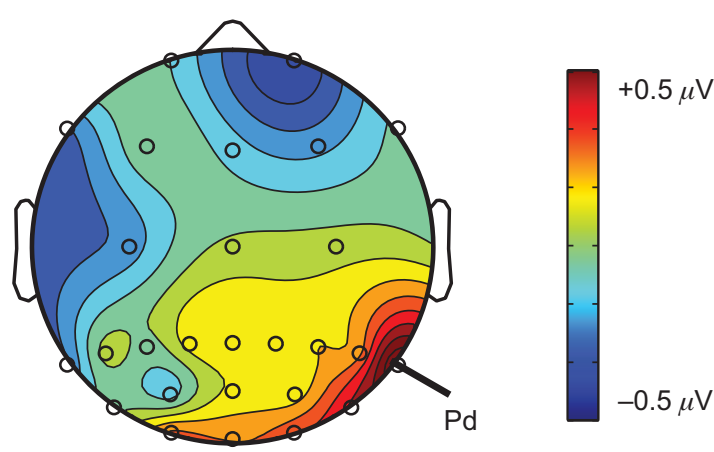

Figure 7. Topographic maps from Experiment 4 for each stimulus category. The top and middle maps show the topography of the N2pc component for targets and target-similar distractors, respectively, measured as the mean amplitude between 225 and 300 msec. The bottom map shows the topography of the Pd component for salient distractors measured as the mean amplitude between 115 and $225 \mathrm{msec}$. The data are arranged so that the left and right sides of the outline head represent ipsilateral and contralateral electrode sites, respectively.

It is, of course, possible that other mechanisms of attention are automatically captured by salient singletons. The mechanism of attention indexed by the $\mathrm{N} 2 \mathrm{pc}$ component is observed whenever participants must bind or localize visual features in the presence of distractors (Luck \& Hillyard, 1994b), and it operates within intermediate and high levels of the ventral pathway (Hopf et al., 2004; Hopf et al., 2000). If some other variety of attention is captured by salient singletons, this variety of attention presumably operates at some other stage of processing.

Although salient singletons do not inevitably capture attention, the present results provide strong evidence that they are nonetheless automatically detected by the visual system. That is, the mere presence of a significant volt- age deflection contralateral to the irrelevant singletons indicates that these singletons were detected by the brain. Moreover, if the association between this contralateral positivity and attentional suppression that was proposed by Hickey et al. (2009) continues to be supported by future research, the present results suggest that the singletons not only were detected, but also triggered an attend-to-me signal and were then subjected to active suppression so that they would not capture attention. It should be emphasized that the Pd component does not directly reflect the attendto-me signal, because this component can be elicited not only by salient distractors, but also by nonsalient distractors $^{4}$ (Hickey et al., 2009). However, the presence of this electrophysiological signature of active suppression sug- 
gests that some kind of attend-to-me signal was generated that required active suppression.

The present results converge with previous behavioral results suggesting that an active suppression process is used to prevent the capture of attention by salient irrelevant singletons (Geyer et al., 2008; Lamy \& Egeth, 2003; Lamy et al., 2004; Theeuwes \& Burger, 1998). Note that the behavioral and electrophysiological results have complementary strengths and weaknesses. Whereas the behavioral studies drew inferences about suppression of the singleton on the basis of changes in target detection performance, which is necessarily indirect, the present electrophysiological signals directly reflect the processing of the irrelevant singleton itself. However, the Pd component used as a measure of suppression in the present study lacks the decades of validation that support RT measures. Note that these very different behavioral and electrophysiological measures converge on the same conclusion-namely, that an active suppression process is used to prevent the capture of attention by salient irrelevant singletons.

On the basis of these converging results, we propose the following hypothesis. Consistent with the bottom-up saliency hypothesis, we propose that salient singletons are automatically detected by the visual system, irrespective of their match with attentional control settings. The only exception to this is when spatial attention is strongly focused on a different region of the display. We further propose that the detection of a salient singleton triggers an attend-to-me signal, which is sent to the attentional control system and will, in the absence of top-down control, induce a shift of attention to the location of the singleton, regardless of whether it matches the attentional control settings. However, consistent with the contingent involuntary orienting hypothesis, we propose that these attentional control settings play a role in determining whether attention is actually shifted to the singleton. If the singleton matches the target template (either in terms of its features or by virtue of being a singleton when singleton-detection mode is being used), attention will in fact be allocated to the singleton. If the singleton does not match the target template, a top-down suppression mechanism may be invoked that prevents attention from being captured by the singleton. However, this suppression mechanism is not automatic but instead requires substantial top-down control, which is presumably mediated by the prefrontal cortex and depends on the availability of working memory resources. If this suppression mechanism is not invoked, attention will be captured by the singleton, regardless of whether it matches the attentional control settings. We call this the signal suppression hypothesis of controlled attention capture.

Whereas behavioral studies of attention capture necessarily present the salient irrelevant singleton and the target in the same display, the irrelevant singleton was never presented in the same array as the target in the present study. Although this simplifies the experimental design and allows the processing of the irrelevant singleton to be assessed in the absence of competition from the target, it leads to one small complication in interpreting the results. Specifically, because the participants knew that the target was never present in the same arrays as the irrelevant singleton, the irrelevant singleton provided some information about the presence or absence of the target. It is therefore possible, in principle, that the participants actually attended to the irrelevant singletons and that the Pd component reflected this allocation of attention. However, this is very unlikely. First, if the irrelevant singletons were attended, an N2pc component should have been observed rather than the Pd component. Second, the irrelevant singletons elicited a Pd component when they appeared inside the to-be-unattended area, as well as inside the to-be-attended area in Experiment 1. We know that attention was restricted to the to-be-attended area in this experiment, because the N2pc was observed for targets and target-similar distractors only when they appeared within this area. Thus, if the Pd component observed for the salient distractors was a result of their implicit task relevance, it should also have been present only for stimuli presented within the to-be-attended area. Thus, although the design of the experiment made it possible that the participants would intentionally focus attention on the salient distractors, the pattern of results indicates that they did not in fact do so. It would be useful for future research to rule out this possibility definitively by removing any correlation between the presence or absence of the irrelevant singleton and the presence or absence of the target.

In addition, one might argue that the salient distractors did not need to be suppressed, because the targets were never presented together with the salient distractors in the present study. However, because the SOA was so short (800-900 msec), this task required a very focused attentional state. If attention had been deployed toward the salient distractors, it would have been more difficult for the participants to maintain this focus of attention, and targets in the subsequent stimulus array may have been missed. Thus, suppression of the irrelevant singletons would have been useful even though they did not appear simultaneously with the targets. In addition, suppression of the irrelevant singletons may have been useful in allowing the participants to avoid making eye movements toward these stimuli.

Several previous ERP studies have not shown a Pd component for salient irrelevant singletons, but this can be explained by the use of tasks that may have encouraged the participants to adopt singleton-detection mode. The study of Hickey et al. (2006), for example, explicitly required participants to detect singleton-defined targets (i.e., circle-among-square trials intermixed with squareamong-circle trials), and in this case an N2pc component was observed for irrelevant color singletons, as well as for target singletons. Other researchers did not use singletondefined targets per se but did use targets that were singletons (Eimer \& Kiss, 2008; Kiss et al., 2008; Rodríguez Holguín et al., 2009). This may have led the participants to adopt singleton-detection mode on at least a subset of trials, and indeed a small but significant N2pc component was observed for irrelevant singletons by Kiss et al. and by Rodríguez Holguín et al. This can also explain the lack of a $\mathrm{Pd}$ component for nontarget singleton stimuli in previous studies in which target arrays contained a singleton in one 
dimension and nontarget arrays contained singletons in other dimensions (e.g., Luck \& Hillyard, 1994b; Schubö \& Müller, 2009). Note that some previous studies showed an early contralateral positivity prior to the N2pc component for targets or target-similar distractors; however, follow-up studies have shown that this effect was a result of the lowlevel sensory imbalance caused by the presence of a single item that contained a rarely occurring feature value among many items that contained a frequently occurring feature value (Leblanc et al., 2008; Luck \& Hillyard, 1994a). The key factor in creating the sensory imbalance may not be the presence of a unique feature value within the array, per se, but, rather, the frequent repetition across trials of the feature value contained by the nonsingleton items, which leads to sensory adaptation for this feature value (see Experiment 4 in Luck \& Hillyard, 1994a).

A Pd component was observed by Eimer and Kiss (2008) for irrelevant color singletons in a cue array that preceded the target array. However, because the Pd component had not yet been characterized when that article was published, Eimer and Kiss did not discuss the possibility of an active suppression process. In addition, they did not rule out the possibility that this effect was a lowlevel sensory effect due to the sensory imbalance caused by the salient irrelevant singleton. This possibility was ruled out in Experiment 3 of the present study, and the finding of a Pd component both in the present study and in the study of Eimer and Kiss demonstrates that this result can be replicated when care is taken to discourage the use of an attentional set that favors the allocation of attention to the irrelevant singleton.

In summary, the present study suggests that salient singletons generate an attention capture signal, irrespective of attentional control settings, but that the deployment of attention to these singletons can be avoided by an active suppression process. Thus, the bottom-up saliency hypothesis is correct insofar as it proposes that salient stimuli are automatically noticed and will, in the absence of top-down control, attract attention even if they do not match the attentional set. However, the contingent involuntary orienting hypothesis is correct insofar as it proposes that attention is not inevitably captured by salient singletons. More broadly, theories of bottom-up and top-down attentional control must include active suppression mechanisms, and these mechanisms must be considered as potential explanations for experiments in which no evidence of attention capture is observed.

\section{AUTHOR NOTE}

This study was supported by Grants R01 MH076226 and R01 MH65034 from the National Institute of Mental Health and by the Japan Society for the Promotion of Science. We gratefully acknowledge the assistance of Javier Lopez-Calderon in data analysis and Alexis Norausky in data collection. Correspondence concerning this article should be addressed to R. Sawaki, University of California Davis, Center for Mind and Brain, 267 Cousteau Place, Davis, CA 95618 (e-mail: rsawaki@ucdavis.edu).

\section{REFERENCE}

American Electroencephalographic Society (1994). Guidelines for standard electrode position nomenclature. Journal of Clinical Neurophysiology, 11, 111-113.
Bacon, W. F., \& Egeth, H. E. (1994). Overriding stimulus-driven attentional capture. Perception \& Psychophysics, 55, 485-496.

Barrett, L. F., Tugade, M. M., \& Engle, R. W. (2004). Individual differences in working memory capacity and dual-process theories of the mind. Psychological Bulletin, 130, 553-573.

Belopolsky, A. V., Zwaan, L., Theeuwes, J., \& Kramer, A. F. (2007). The size of an attentional window modulates attentional capture by color singletons. Psychonomic Bulletin \& Review, 14, 934-938.

Corbetta, M., \& Shulman, G. L. (2002). Control of goal-directed and stimulus-driven attention in the brain. Nature Reviews Neuroscience, 3, 201-215.

Cosman, J. D., \& Vecera, S. P. (2009). Perceptual load modulates attentional capture by abrupt onsets. Psychonomic Bulletin \& Review, 16, 404-410.

Delorme, A., \& MAKeIG, S. (2004). EEGLAB: An open source toolbox for analysis of single-trial EEG dynamics including independent component analysis. Journal of Neuroscience Methods, 134, 9-21.

Desimone, R., \& Duncan, J. (1995). Neural mechanisms of selective visual attention. Annual Review of Neuroscience, 18, 193-222.

Desimone, R., \& Schein, S. J. (1987). Visual properties of neurons in area V4 of the macaque: Sensitivity to stimulus form. Journal of Neurophysiology, 57, 835-868.

Egeth, H. E., \& Yantis, S. (1997). Visual attention: Control, representation, and time course. Annual Review of Psychology, 48, 269-297.

EIMER, M. (1996). The N2pc component as an indicator of attentional selectivity. Electroencephalography \& Clinical Neurophysiology, 99, 225-234.

EImer, M., \& Kiss, M. (2008). Involuntary attentional capture is determined by task set: Evidence from event-related brain potentials. Journal of Cognitive Neuroscience, 20, 1423-1433.

Eimer, M., Kiss, M., Press, C., \& SAUter, D. (2009). The roles of feature-specific task set and bottom-up salience in attentional capture: An ERP study. Journal of Experimental Psychology: Human Perception \& Performance, 35, 1316-1328.

FolK, C. L., Leber, A. B., \& Egeth, H. E. (2002). Made you blink! Contingent attentional capture produces a spatial blink. Perception \& Psychophysics, 64, 741-753.

Folk, C. L., \& Remington, R. [W.] (1998). Selectivity in distraction by irrelevant featural singletons: Evidence for two forms of attentional capture. Journal of Experimental Psychology: Human Perception \& Performance, 24, 847-858.

FolK, C. L., \& Remington, R. [W.] (1999). Can new objects override attentional control settings? Perception \& Psychophysics, 61, 727-739.

FolK, C. L., Remington, R. W., \& Johnston, J. C. (1992). Involuntary covert orienting is contingent on attentional control settings. Journal of Experimental Psychology: Human Perception \& Performance, 18, 1030-1044.

Folk, C. L., Remington, R. W., \& Wright, J. H. (1994). The structure of attentional control: Contingent attentional capture by apparent motion, abrupt onset, and color. Journal of Experimental Psychology: Human Perception \& Performance, 20, 317-329.

Found, A., \& Müller, H. J. (1996). Searching for unknown feature targets on more than one dimension: Investigating a "dimensionweighting" account. Perception \& Psychophysics, 58, 88-101.

FuKUDA, K., \& Vogel, E. K. (2009). Human variation in overriding attentional capture. Journal of Neuroscience, 29, 8726-8733.

Geyer, T., Müller, H. J., \& Krummenacher, J. (2008). Expectancies modulate attentional capture by salient color singletons. Vision Research, 48, 1315-1326.

HeInZE, H. J., \& MANGUN, G. R. (1995). Electrophysiological signs of sustained and transient attention to spatial locations. Neuropsychologia, 33, 889-908.

Hickey, C., Di Lollo, V., \& McDonald, J. J. (2009). Electrophysiological indices of target and distractor processing in visual search. Journal of Cognitive Neuroscience, 21, 760-775.

Hickey, C., McDonald, J. J., \& Theeuwes, J. (2006). Electrophysiological evidence of the capture of visual attention. Journal of Cognitive Neuroscience, 18, 604-613.

Hilimire, M. R., Mounts, J. R., Parks, N. A., \& Corballis, P. M. (2009). Competitive interaction degrades target selection: An ERP study. Psychophysiology, 46, 1080-1089.

Hopf, J. M., Boelmans, K., Schoenfeld, M. A., Luck, S. J., \& Heinze, H. J. (2004). Attention to features precedes attention to 
locations in visual search: Evidence from electromagnetic brain responses in humans. Journal of Neuroscience, 24, 1822-1832.

Hopf, J. M., Luck, S. J., Girelli, M., Hagner, T., Mangun, G. R., Scheich, H., \& HeInZE, H. J. (2000). Neural sources of focused attention in visual search. Cerebral Cortex, 10, 1233-1241.

Johannes, S., Münte, T. F., Heinze, H. J., \& Mangun, G. R. (1995). Luminance and spatial attention effects on early visual processing. Cognitive Brain Research, 2, 189-205.

KastNer, S., \& Ungerleider, L. G. (2000). Mechanisms of visual attention in the human cortex. Annual Review of Neuroscience, 23, 315-341.

Kiss, M., Jolicceur, P., Dell'Acqua, R., \& Eimer, M. (2008). Attentional capture by visual singletons is mediated by top-down task set: New evidence from the N2pc component. Psychophysiology, 45, 1013-1024.

KNIERIM, J. J., \& VAN Essen, D. C. (1992). Neuronal responses to static texture patterns in area V1 of the alert macaque monkey. Journal of Neurophysiology, 67, 961-980.

Lamy, D., \& EgETh, H. E. (2003). Attentional capture in singletondetection and feature-search modes. Journal of Experimental Psychology: Human Perception \& Performance, 29, 1003-1020.

LAMY, D., Leber, A., \& EGETH, H. E. (2004). Effects of task relevance and stimulus-driven salience in feature-search mode. Journal of Experimental Psychology: Human Perception \& Performance, 30, 1019-1031.

Lavie, N. (1995). Perceptual load as a necessary condition for selective attention. Journal of Experimental Psychology: Human Perception \& Performance, 21, 451-468.

LaVIE, N. (2005). Distracted and confused? Selective attention under load. Trends in Cognitive Sciences, 9, 75-82.

LAVIE, N., \& DE FOCKERT, J. (2005). The role of working memory in attentional capture. Psychonomic Bulletin \& Review, 12, 669-674.

Lavie, N., Hirst, A., De Fockert, J. W., \& Viding, E. (2004). Load theory of selective attention and cognitive control. Journal of Experimental Psychology: General, 133, 339-354.

LAVIE, N., \& TsaL, Y. (1994). Perceptual load as a major determinant of the locus of selection in visual attention. Perception \& Psychophysics, 56, 183-197.

Leber, A. B., \& Egeth, H. E. (2006). It's under control: Top-down search strategies can override attentional capture. Psychonomic Bulletin \& Review, 13, 132-138.

Leblanc, E., Prime, D. J., \& Jolicceur, P. (2008). Tracking the location of visuospatial attention in a contingent capture paradigm. Journal of Cognitive Neuroscience, 20, 657-671.

Lien, M. C., Ruthruff, E., Goodin, Z., \& Remington, R. W. (2008). Contingent attentional capture by top-down control settings: Converging evidence from event-related potentials. Journal of Experimental Psychology: Human Perception \& Performance, 34, 509-530.

Lins, O. G., Picton, T. W., Berg, P., \& Scherg, M. (1993). Ocular artifacts in EEG and event-related potentials. I: Scalp topography. Brain Topography, 6, 51-63.

Lu, S., \& HaN, S. (2009). Attentional capture is contingent on the interaction between task demand and stimulus salience. Attention, Perception, \& Psychophysics, 71, 1015-1026.

LUCK, S. J. (1995). Multiple mechanisms of visual-spatial attention: Recent evidence from human electrophysiology. Behavioural Brain Research, 71, 113-123.

LuCK, S. J., FAn, S., \& Hillyard, S. A. (1993). Attention-related modulation of sensory-evoked brain activity in a visual search task. Journal of Cognitive Neuroscience, 5, 188-195.

LUCK, S. J., \& ForD, M. A. (1998). On the role of selective attention in visual perception. Proceedings of the National Academy of Sciences, 95, 825-830.

Luck, S. J., Fuller, R. L., Braun, E. L., Robinson, B., SummerFELT, A., \& GolD, J. M. (2006). The speed of visual attention in schizophrenia: Electrophysiological and behavioral evidence. Schizophrenia Research, 85, 174-195.

Luck, S. J., Girelli, M., McDermot, M. T., \& Ford, M. A. (1997). Bridging the gap between monkey neurophysiology and human perception: An ambiguity resolution theory of visual selective attention. Cognitive Psychology, 33, 64-87.

LUCK, S. J., \& HiLlYARD, S. A. (1994a). Electrophysiological correlates of feature analysis during visual search. Psychophysiology, 31, 291-308.

LuCK, S. J., \& Hillyard, S. A. (1994b). Spatial filtering during visual search: Evidence from human electrophysiology. Journal of Experimental Psychology: Human Perception \& Performance, 20, 10001014.

Luck, S. J., Hillyard, S. A., Mouloua, M., WoldorfF, M. G., Clark, V. P., \& Hawkins, H. L. (1994). Effects of spatial cuing on luminance detectability: Psychophysical and electrophysiological evidence for early selection. Journal of Experimental Psychology: Human Perception \& Performance, 20, 887-904.

Mangun, G. R., \& Hillyard, S. A. (1988). Spatial gradients of visual attention: Behavioral and electrophysiological evidence. Electroencephalography \& Clinical Neurophysiology, 70, 417-428.

Mazza, V., Turatto, M., \& Caramazza, A. (2009). An electrophysiological assessment of distractor suppression in visual search tasks. Psychophysiology, 46, 771-775.

Mazza, V., Turatto, M., Umiltà, C., \& Eimer, M. (2007). Attentional selection and identification of visual objects are reflected by distinct electrophysiological responses. Experimental Brain Research, 181, 531-536.

Müller, H. J., Heller, D., \& Ziegler, J. (1995). Visual search for singleton feature targets within and across feature dimensions. Perception \& Psychophysics, 57, 1-17.

Munneke, J., Van der Stigchel, S., \& Theeuwes, J. (2008). Cueing the location of a distractor: An inhibitory mechanism of spatial attention? Acta Psychologica, 129, 101-107.

Rodríguez Holguín, S., Doallo, S., Vizoso, C., \& Cadaveira, F. (2009). N2pc and attentional capture by colour and orientationsingletons in pure and mixed visual search tasks. International Journal of Psychophysiology, 73, 279-286.

RufF, C. C., \& Driver, J. (2006). Attentional preparation for a lateralized visual distractor: Behavioral and fMRI evidence. Journal of Cognitive Neuroscience, 18, 522-538.

Sawaki, R., \& Katayama, J. (2008). Top-down directed attention to stimulus features and attentional allocation to bottom-up deviations. Journal of Vision, 8(15, Art. 4), 1-8.

SaWAKi, R., \& Katayama, J. (2009). Difficulty of discrimination modulates attentional capture by regulating attentional focus. Journal of Cognitive Neuroscience, 21, 359-371.

SCHUBÖ, A., \& MüLLER, H. J. (2009). Selecting and ignoring salient objects within and across dimensions in visual search. Brain Research, 1283, 84-101.

Seiss, E., Kiss, M., \& Eimer, M. (2009). Does focused endogenous attention prevent attentional capture in pop-out visual search? Psychophysiology, 46, 703-717.

Slotnick, S. D., Schwarzbach, J., \& Yantis, S. (2003). Attentional inhibition of visual processing in human striate and extrastriate cortex. Neurolmage, 19, 1602-1611.

Theeuwes, J. (1991a). Cross-dimensional perceptual selectivity. Perception \& Psychophysics, 50, 184-193.

TheEuwes, J. (1991b). Exogenous and endogenous control of attention: The effect of visual onsets and offsets. Perception \& Psychophysics, 49, 83-90.

Theeuwes, J. (1992). Perceptual selectivity for color and form. Perception \& Psychophysics, 51, 599-606.

Theeuwes, J. (1994). Stimulus-driven capture and attentional set: Selective search for color and visual abrupt onsets. Journal of Experimental Psychology: Human Perception \& Performance, 20, 799-806.

THEEUWES, J. (2004). Top-down search strategies cannot override attentional capture. Psychonomic Bulletin \& Review, 11, 65-70.

Theeuwes, J., Atchley, P., \& Kramer, A. F. (2000). On the time course of top-down and bottom-up control of visual attention. In S. Monsell $\&$ J. Driver (Eds.), Control of cognitive processes: Attention and performance XVIII (pp. 105-124). Cambridge, MA: MIT Press.

Theeuwes, J., \& Burger, R. (1998). Attentional control during visual search: The effect of irrelevant singletons. Journal of Experimental Psychology: Human Perception \& Performance, 24, 1342-1353.

Theeuwes, J., Kramer, A. F., \& Atchley, P. (2001). Spatial attention in early vision. Acta Psychologica, 108, 1-20.

Vecera, S. P., \& LucK, S. J. (2002). Attention. In V. S. Ramachandran (Ed.), Encyclopedia of the human brain (pp. 269-284). San Diego: Academic Press.

Vogel, E. K., McCollough, A. W., \& Machizawa, M. G. (2005). Neural measures reveal individual differences in controlling access to working memory. Nature, 438, 500-503. 
Woodman, G. F., \& LUCK, S. J. (1999). Electrophysiological measurement of rapid shifts of attention during visual search. Nature, $\mathbf{4 0 0}$, 867-869.

Woodman, G. F., \& Luck, S. J. (2003). Serial deployment of attention during visual search. Journal of Experimental Psychology: Human Perception \& Performance, 29, 121-138.

YANTIS, S., \& EgETH, H. E. (1999). On the distinction between visual salience and stimulus-driven attentional capture. Journal of Experimental Psychology: Human Perception \& Performance, 25, 661-676.

YANTIS, S., \& Jonides, J. (1990). Abrupt visual onsets and selective attention: Voluntary versus automatic allocation. Journal of Experimental Psychology: Human Perception \& Performance, 16, 121-134.

\section{NOTES}

1. The present study is focused on salience that is determined by the interrelationships among the items in the scene (i.e., a letter of one color among letters of another color), rather than by the intrinsic properties of the individual items. The capture of attention by the intrinsic property of an individual item (e.g., an onset) may follow a different set of rules.

2. In most behavioral studies of attention capture, the irrelevant singleton is presented simultaneously with the target, and capture of attention is assessed through the effect of the irrelevant singleton on target-detection performance. Because ERP recordings make it possible to assess the processing of the irrelevant singleton itself, the irrelevant singleton in the present study was not presented in the same array as the target. This greatly simplifies the experimental design and makes it possible to assess the capture of attention by the irrelevant singleton uncontaminated by competition from a simultaneous target.

3. When the array contained an item with the target letter identity and target letter size in the unattended region, we refer to this as a target within the unattended area. However, the participants were instructed not to respond to these stimuli.

4. It might seem problematic that the salient singleton elicited a Pd component in the present study, whereas a relatively nonsalient distractor elicited a Pd component in the study of Hickey et al. (2009). However, in the Hickey et al. (2009) study, the target and the distractor were the only items present in the display. Under such low-load conditions, relatively nonsalient items can provide strong competition for attention (Lavie, 1995, 2005; Lavie, Hirst, de Fockert, \& Viding, 2004; Lavie \& Tsal, 1994). Thus, active suppression may be needed for high-salience distractors under high-load conditions (as in the present study) and also for low-salience distractors under low-load conditions (as in the study of Hickey et al., 2009).

(Manuscript received October 15, 2009; revision accepted for publication March 21, 2010.) 